\title{
Rotationally Variant Grating Writing in Photonic Crystal Fibres
}

\author{
J. Holdsworth ${ }^{*}, 1,2$, K. Cook ${ }^{2}$, J. Canning ${ }^{2}$, S. Bandyopadhyay ${ }^{2,3}$ and M. Stevenson ${ }^{2}$ \\ ${ }^{I}$ School of Mathematical and Physical Sciences, University of Newcastle, Callaghan NSW, 2308, Australia \\ ${ }^{2}$ Interdisciplinary Photonics Laboratories (iPL), School of Chemistry, University of Sydney, NSW, 2006 Australia \\ ${ }^{3}$ Central Glass and Ceramic Research Institute, 196 Raja S C Mullick Road, Kolkata, 700032 India
}

\begin{abstract}
The role of rotational alignment during grating writing is comprehensively examined by the measurement of core luminescence from a Ge-doped 12 ring photonic crystal fibre, computer simulation of transmission and, definitively, by inscription of gratings at three angles- $0^{\circ}, 30^{\circ}$ and the optimum luminescent angle for this fibre, $21.5^{\circ}$. All experimental results were in accordance with each other and in agreement with simulation. Control of the rotational alignment of the fibre is demonstrably critical for reproducible grating writing.
\end{abstract}

Keywords: Fibre bragg gratings, microstructured fibres, photonic crystal fibres.

\section{INTRODUCTION}

The rotational symmetry within structured optical fibres such as suspended core fibres [1], photonic crystal fibres [2] and Fresnel fibres [3], introduces a challenge to grating writing not present when using conventional rotationally invariant solid fibres. High levels of scattered light arise from multiple interface reflections within the hole array and vary as the structure is rotated under illumination. This scatter carries information regarding the fibre structure and this property has been used as a photonic crystal filter [4] and for signal encryption [5]. For grating writing, the variation of light reaching the core can potentially determine whether grating writing within structured fibres succeeds or not. An obvious solution to overcome scatter is to temporarily fill the holes with index matching fluids [6,7]. However, this approach is restricted to wavelengths above the band edge of the fibre material and is increasingly problematic for shorter wavelengths. For $193 \mathrm{~nm}$ appropriate index-matching solutions are not readily available and, in addition, higher-order photon effects both in the UV and in the near IR can cause significant heating of the fluids, if they are too close to the fibre core, which can damage the fibre.

In spite of this scatter, Bragg grating writing has been demonstrated in various structured fibres including: (a) photonic crystal fibres with photosensitive germanosilicate cores, with and without hydrogen using single photon excitation [8-11]; (b) in $\mathrm{Er}^{3+}$-doped aluminosilicate core photonic crystal fibres using two-photon excitation $[12,13]$; and (c) in pure single material silica fibres also using two-photon excitation $[14,15]$ and higher exponent femtosecond laser inscription [16]. However, the scatter of light incident from the side of the fibre does cause variation in the intensity within the fibre core during grating writing, both through incoherent scattering and coherent interference. This poses significant challenges to grating reproducibility. In the case of higher

*Address correspondence to this author at the School of Mathematical and Physical Sciences, University of Newcastle, Callaghan NSW, 2308, Australia; E-mail: john.holdsworth@newcastle.edu.au exponent processes, this variation is sufficient to mark the difference between successful and unsuccessful grating writing. Greater control of the writing process is required.

In this paper, the implications of the variance in rotational scatter on conventional, single UV photon grating writing within a structured fibre containing 12 rings are considered. The measured UV-induced luminescence from typeII [17] germanosilicate oxygen deficient centre (Ge-ODC) defects, further classified as luminescence defects [18], within the germanium-doped fibre core is compared with results obtained for actual grating writing in the fibre. Numerical simulation is also used to further elaborate the origin of the particular optimum angle observed.

\section{FIBRE AND FDTD SIMULATION}

The fibre used in this work is a commercially sourced 12ring photonic crystal fibre (Crystal Fibre). A scanning electron microscope (SEM) image of the cross-section is shown in Fig. (1a). It has a $33 \mu \mathrm{m}$ triangular lattice of holes ( $\phi_{\text {hole diam }}=0.625 \mu \mathrm{m}$, pitch $\left.\Lambda=1.44 \mu \mathrm{m}\right)$ with a triangular core made up of four defects. In the centre of this core is a photosensitive step-index region $\left(\phi_{\text {core diam }}=3 \mu \mathrm{m}\right)$ of germanosilicate glass within the overall diameter $\left(\phi_{\text {fibre diam }}=120 \mu \mathrm{m}\right)$. The fibre is single mode at $1550 \mathrm{~nm}$. Both Type I and Type IIa gratings have been recently demonstrated [7] in this fibre without rotational alignment of the lattice.

Type I grating inscription requires the core of the fibre be illuminated with sufficient power intensity to induce the required refractive index change within the germanosilicate core in spite of the scattering of light from the lattice. Computer simulation using finite difference time domain (FDTD) has been used to study and anticipate the dependence on rotation. To date, simulations of an idealized four-ring structure into which gratings are typically inscribed using a free space interferometric system or direct writing through an optical phase mask, have been done with ray trace software and with FDTD software. Contradictory results were observed given the limitations in the ray trace analysis, but 
both highlighted the role of scattering to the grating writing process.
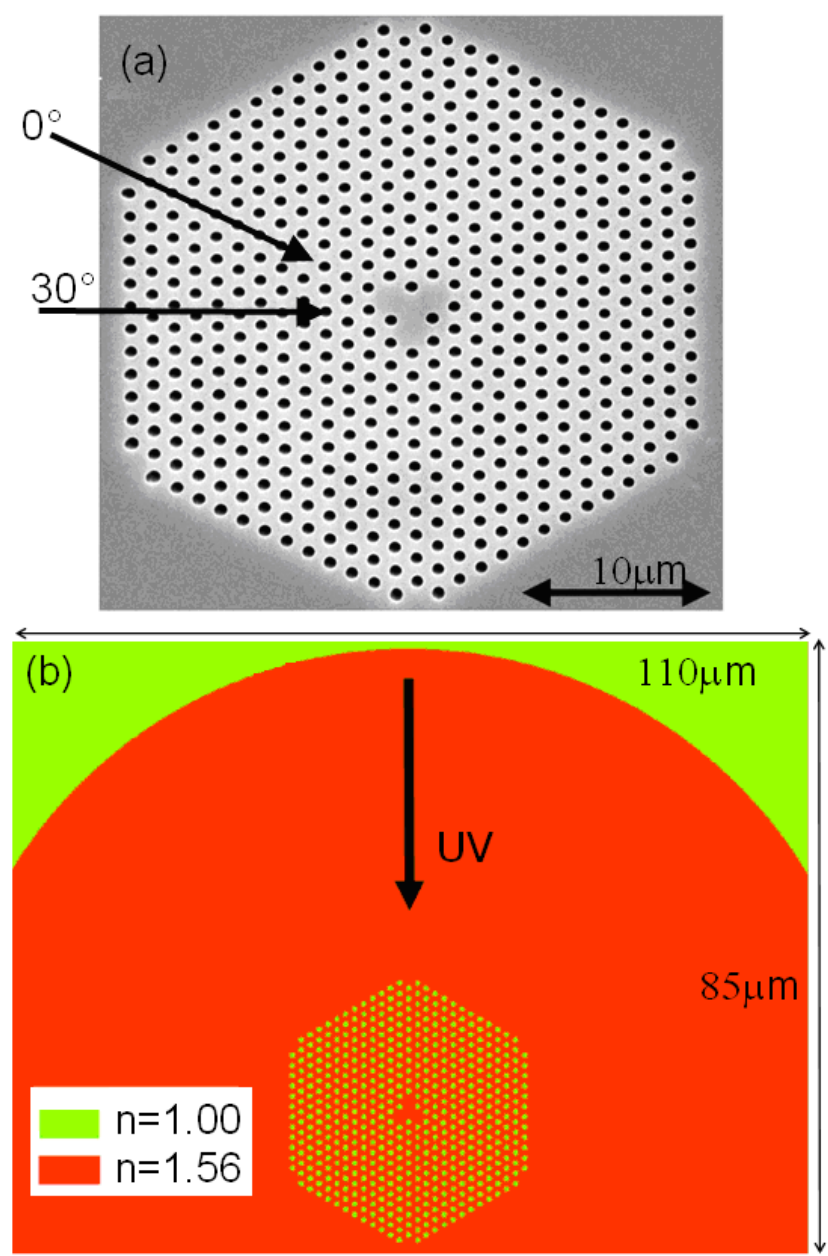

Fig. (1). (a) SEM micrograph of Crystal Fibre NL-1550 12-ring photonic crystal fibre (PCF) and (b) Model framework for simulation showing the $0^{\circ}$ or $\Gamma \mathrm{K}$ direction, the UV light writing light is incident from above.

An IR multi-photon grating inscription model [19] where refractive index change in the core glass is induced by very tightly focused femtosecond duration IR light pulses, similarly highlighted the importance of scatter. These simulations were performed under a very different writing conditions than the plane wave CW $244 \mathrm{~nm}$ excitation or the approximate plane wave $193 \mathrm{~nm}$ ArF source illumination achieved at the $\sim 40 \mu \mathrm{m}$ FWHM cylindrical lens focus employed in this work. Recent work in a highly birefringent fibre [20] with only one layer of holes did not exhibit a significant rotational dependence, which is expected given the few interface layers involved.

A commercially available FDTD software package (Optiwave Corporation) is used in this work to better understand the angular dependence of the light reaching the core. Fig. (1b) shows the model framework used in the simulation based on the SEM image of Fig. (1a). A plane wavefront of $193 \mathrm{~nm}$ radiation is incident from the top of Fig. (1b) with a Gaussian spatial profile ( $40 \mu \mathrm{m}$ FWHM) and a fluence of $500 \mathrm{~mJ} / \mathrm{cm}^{2}$, consistent with typical writing conditions. The simulation grid mesh size is $30 \mathrm{~nm}$. In this 2-D simulation, only the orthogonal excitation of the structure from the outside was considered despite the diffraction orders from the phase mask having a vectorial component angled $\sim 30-35^{\circ}$ to the fibre axis. The justification for this is the net index change dependence on cumulative fluence and the fact that the bulk of the scatter is dictated by the orthogonal component. The angled component will affect the quality of the fringe pattern at the core rather than the total cumulative fluence.

The resultant contour plots of the electric field amplitude are shown below (Fig. 2) for three angles: $0^{\circ}$ direction, $21.5^{\circ}$ (the angle of greatest experimentally determined fluorescence - see below) and $30^{\circ}$. It can be seen that $21.5^{\circ}$ has the highest amount of light within the photosensitive core at the centre of the triangular core. Integration of the central core region also confirms this with normalized total fields of 0.87 , 0.92 and $1.00 \pm 0.01$ a.u. for the $30^{\circ}, 0^{\circ}$ and $21.5^{\circ}$ cases respectively. It is therefore expected, in the first instance that the largest index change will be obtained at $21.5^{\circ}$ if the index change arises purely from GeODC generated polarisability changes within the photosensitive core alone. For both $0^{\circ}$ and $30^{\circ}$ any induced changes are likely to be identical within experimental error. However, given the very high germanium content, this fibre is susceptible to early type IIa grating formation [7], which inherently depends on corecladding stress changes [21, 22]. A component of index change which may extend beyond the photosensitive core is likely to be present. The experiments reported here using this unusual triangular structured core, provide spatial determination and can in principle be used as an excellent qualitative probe of the contributions of the index change from both defect polarisability and core-cladding stresses.

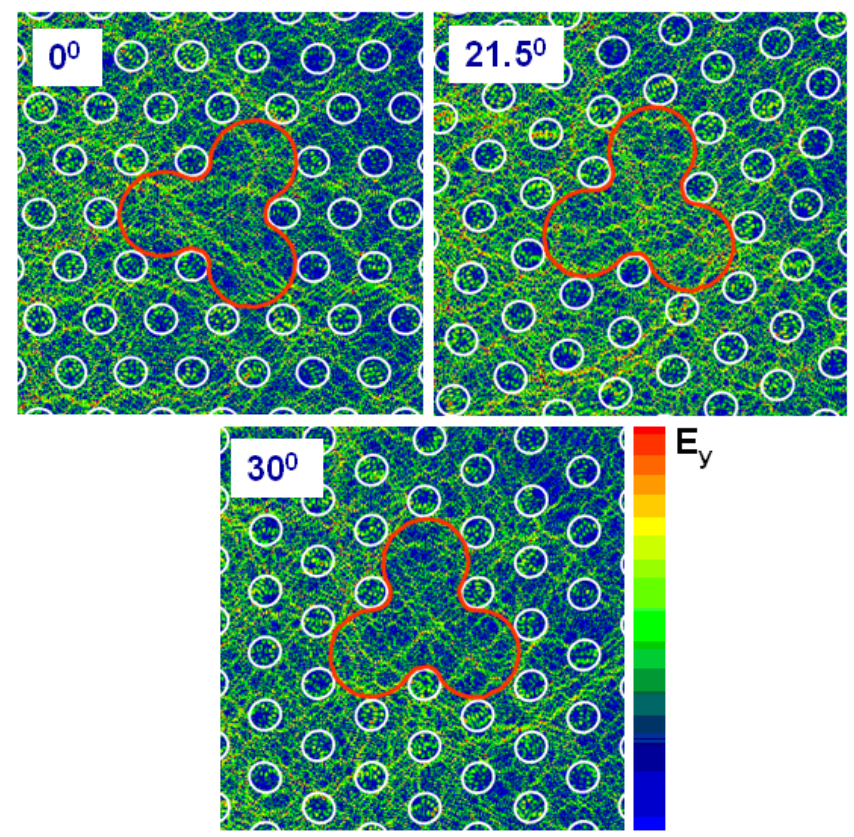

Fig. (2). FDTD simulation output for $193 \mathrm{~nm}$ illumination from the left for $0^{\circ}, 21.5^{\circ}$ and $30^{\circ}$ angles. The UV light is incident from the left. The positions of the air holes are shown in white; the tri-lobed core region is outlined in red for clarity. 


\section{EXPERIMENTAL}

The experimental investigation of illuminating the 12 ring PCF was undertaken with two objectives: (1) to measure the defect-generated luminescence from the centre of the fibre core under $244 \mathrm{~nm}$ excitation and (2) to examine the grating strength achieved when gratings were written into the fibre at different known angles. In contrast to $193 \mathrm{~nm}, 244$ $\mathrm{nm}$ was chosen for this work as the ODC blue luminescence quantum efficiency and continuous vs pulsed illumination yield detectable signal levels. However, grating writing itself is carried out at $193 \mathrm{~nm}$ as this has been shown to be more efficient without hydrogen [10].

\subsection{Defect Luminescence}

The fluorescence experiment matched the grating writing condition of a plane wavefront illuminating the fibre and coupling to the core with the fibre illuminated by $244 \mathrm{~nm}$ UV light from a frequency doubled $\mathrm{Ar}^{+}$laser focused through a cylindrical lens (f.l. $=500 \mathrm{~mm}$ ) to generate blue luminescence from excitation of the $\mathrm{Ge}(\mathrm{ODC})$ defects within the core over a $2 \mathrm{~mm}$ length of fibre. The fibre core was positioned within the Rayleigh range of the lens for approximately parallel, diffraction-free illumination. The fibre was rotated constantly with a motorized rotary actuator and the fluorescence signal was measured on a filtered $\mathrm{Si}$ detector. The end of the fibre was imaged by a CMOS camera, illustrated in Fig. (3).

The average fluorescence signal power recorded was 40 $\mathrm{nW}$ with a rotational variance of $20 \mathrm{nW}(-8 /+12 \mathrm{nW}$ which is -20 to $+30 \%$ variation about the average value with rotation. The measurement had a statistical error per point of \pm $20 \mathrm{pW}$. The excellent regularity of the lattice structure is evident in the regular intervals in the continuous data stream shown in Fig. (4). The fluorescence maxima is observed at $21.5^{\circ}$ which determined the selection of $21.5^{\circ}$ for the simulation described earlier. Generally, it is interesting to comment that overall the variation is insufficient to stop grating writing for single photon grating processes but sufficient to stop two or more photon excitation processes carried out just below the damage threshold. From these experiments, it can be concluded that if the index change is solely arising from defect polarisability changes confined to the photosensitive core alone, then the angle of $21.5^{\circ}$ is the optimum angle for grating writing of Type I gratings in this fibre.

\subsection{Grating Writing}

The angle resolved inscription of a grating remains the definitive test to experimentally determine the optimum angle for grating writing in PCF. The confirmation of any correlation between luminescence at $244 \mathrm{~nm}$ and Type I grating writing at $193 \mathrm{~nm}$ will emerge from analysis of actual written gratings. The experimental arrangement is similar to previously published work [7] with the addition of dual, precision motorized rotary actuators to hold the fibre as shown in Fig. (5). A coupling optimized splice to the PCF's left hand end ensures light is coupled into the fundamental mode of the PCF core. This enables in-situ monitoring of grating growth using a transmitted tunable semiconductor laser $(1 \mathrm{pm}$ resolution). The right hand side of the sample is first imaged by the CMOS camera to set the angle of the fibre core with respect to the illuminating $193 \mathrm{~nm}$ laser light, and then subsequently butt coupled for diagnostic transmission.

Inscription of 9 gratings at three angles, $0^{\circ}, 21.5^{\circ}$ and $30^{\circ}$, was undertaken with the $193 \mathrm{~nm}$ laser fluence into the fibre held constant, within practical limits. The comparative results are shown in Fig. (6). They show that the average index change, $\Delta n_{\mathrm{av}}$, is highest when core is illuminated at $21.5^{\circ}$, in agreement with both the luminescence study and simulation. This agreement is taken as a measure of the con-
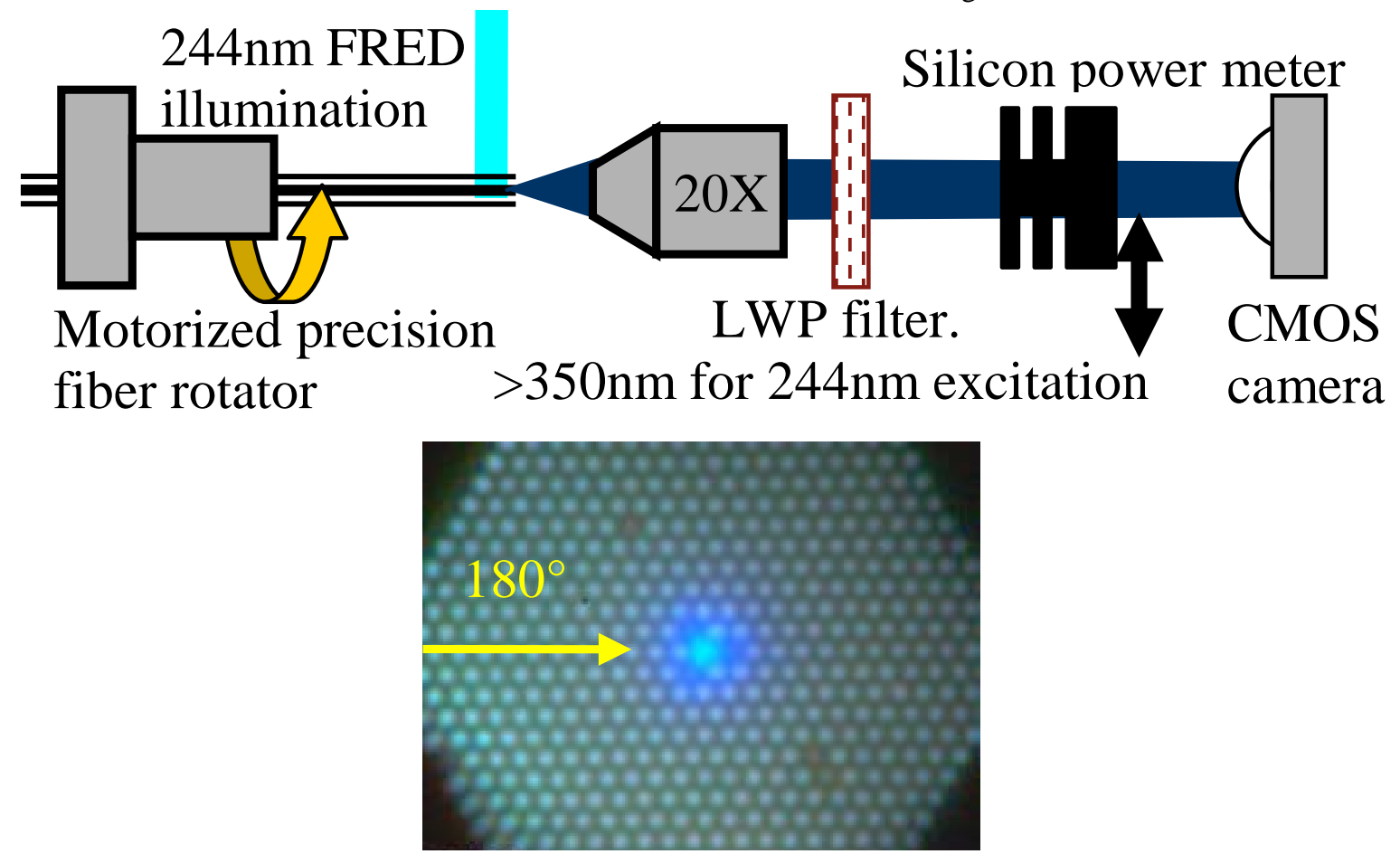

Fig. (3). Schematic of $244 \mathrm{~nm}$ exited Ge-Oxygen deficient site fluorescence experiment with image of fluorescence signal emanating from $244 \mathrm{~nm}$ irradiation along the $\Gamma \mathrm{M}$ direction. 


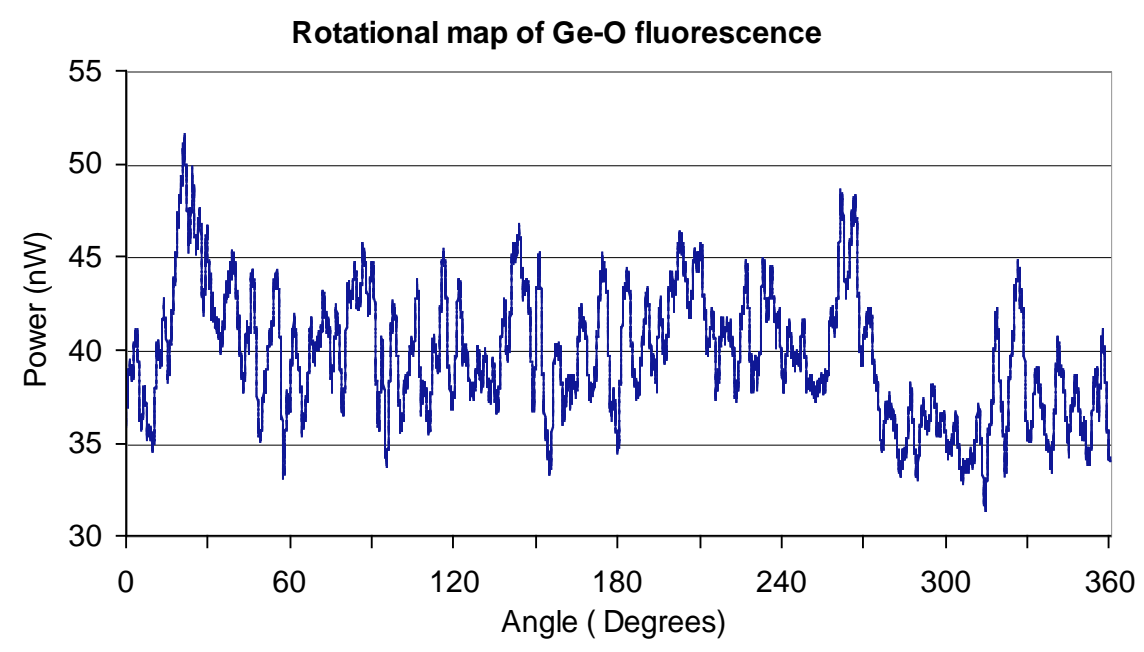

Fig. (4). The longitudinal rotation angular dependence of the $244 \mathrm{~nm}$ induced signal.

tribution to scatter that the 12-ring structure of the fibre engenders.

That said, the definition a type II absorption Ge(ODC, II A) and a type II luminescence $\mathrm{Ge}(\mathrm{ODC}$, II L) [18] does mean the writing wavelength of $193 \mathrm{~nm}$ engages with the higher energy absorption of the $\mathrm{Ge}(\mathrm{ODC}$, II A) site and this work did not measure the alteration in $400 \mathrm{~nm}$ luminescence during the grating writing exposure which has been used as a measure of physical change within the glass [18].

In contrast, the index modulation reflected by the strength of the gratings as shown in Fig. (6b), does show some variance in the evolution of the grating strength with cumulative $193 \mathrm{~nm}$ fluence coupling into the core via the absorption $\mathrm{Ge}(\mathrm{ODC}$, II A). Modal interference or beating can be observed [7] - this fibre also supports a tri-lobed higher order mode which can be excited and beats with the fundamental mode as the average index increases, and therefore phase matching at the spliced output varies accordingly. Unlike the average index, the index modulation is determined by the fringe contrast and the quality of the interference. Therefore, whilst $21.5^{\circ}$ may give the best overall result in terms of the highest amount of $244 \mathrm{~nm}$ and, by inference, $193 \mathrm{~nm}$ light accessing the core, it may in turn be far more sensitive to variations from the orthogonal axis which can give rise to differences in constructive and destructive interference at the core from which the fringe profile is derived. What these results indicate is that not only is the quantity of
UV light reaching the core important for grating growth but so too is the local interference, both constructive and destructive, arising from scattering at all the various interfaces within the coherence of the writing laser $(\sim 400 \mu \mathrm{m})$. This will determine a number of parameters including grating strength, the transverse profile across the core and so on. All of this indicates that alignment is far more critical for reproducible fringe profiles and therefore grating strengths within these fibres than for conventional photosensitive fibres.

Finally we note that the onset "threshold" of Type IIa grating formation is not severely affected by the interference although the grating strength itself is. This suggests that within the resolution of this optical probe, both $\Delta n$ changes for Type I and Type IIa gratings are predominantly within the doped core and at the interface between the doped core and the rest of the core.

\section{CONCLUSIONS}

Simulation, measurement of $400 \mathrm{~nm}$ core luminescence, the onset of type IIa formation, achieved grating strength and average index change have been studied to gauge the impact of scattering within a 12-ring photonic crystal fibre. There is overall consistent agreement between Ge(ODC, II L) luminescence experiments, simulation and, definitively, grating writing to indicate that control of the rotational alignment of the writing beam with respect to the fibre is critical for single and particularly multi-photon inscription processes to ensure

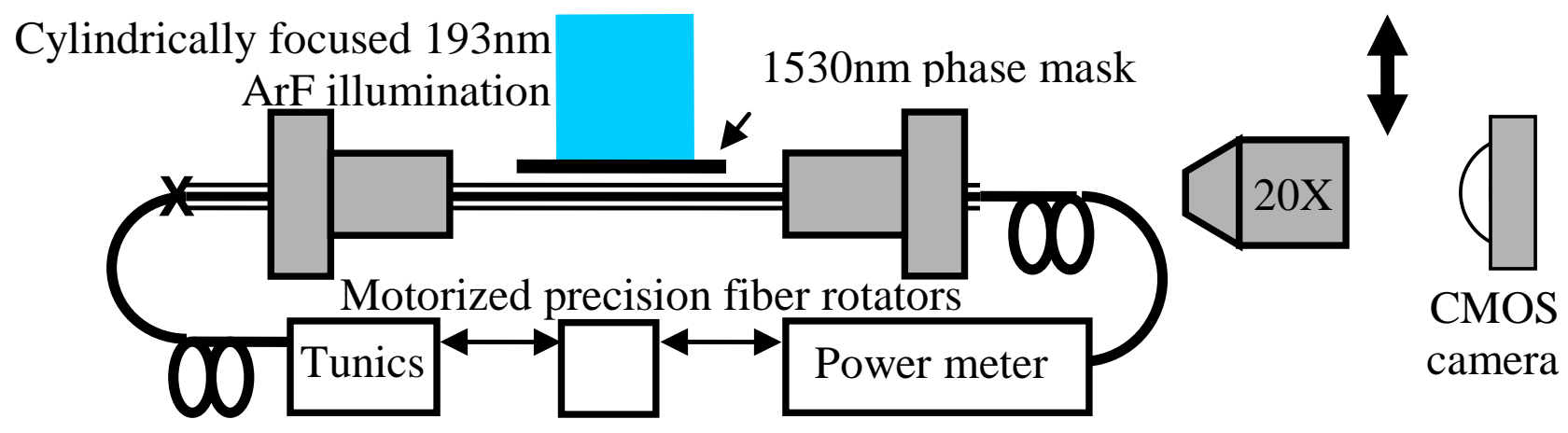

Fig. (5). The rotationally agile grating writing arrangement. 
reproducible quantities of light reach the photosensitive core. The alignment necessary to ensure local scattering induced interference does not affect the holographic fringe visibility required to actually inscribe the periodic structure that determines the grating strength is a significant challenge.
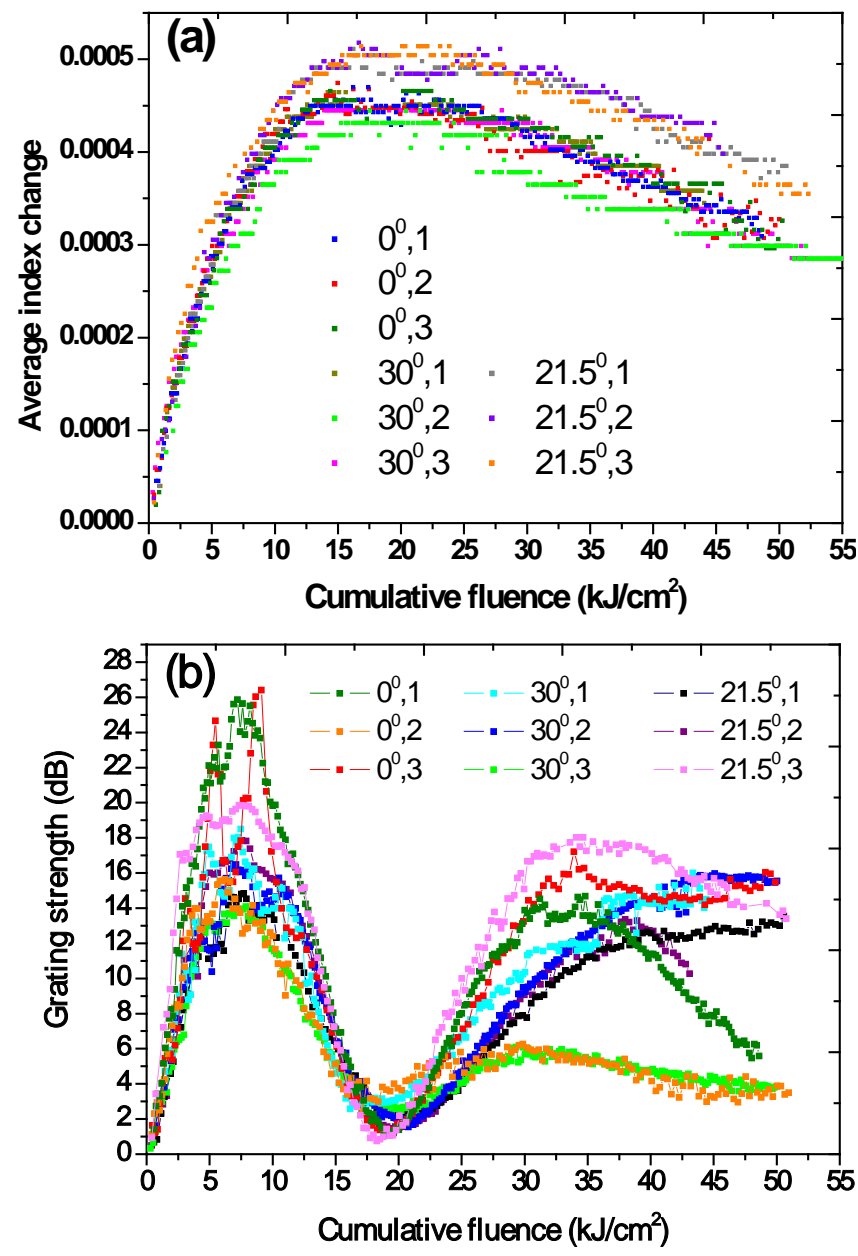

Fig. (6). (a) The cumulative index change in the glass core and (b) the strength of grating written into the PCF at three different illumination angles.

\section{ACKNOWLEDGEMENTS}

Jason De Iuliis is acknowledged for identifying the problem. This work was supported by the University of Newcastle and the Australian Research Council Discovery Projects program. Somnath Bandyopadhyay acknowledges a Raman Fellowship from the Commonwealth Science and Industry Research (CSIR) Agency, India.

\section{REFERENCES}

[1] Kaiser P, Marcatili EAJ, Miller SE. A new optical fiber. Bell Syst Tech J 1973; 52: 265-69.

[2] Bjarklev A, Broeng J, Sanchez Bjarklev A. Photonic crystal fibres. Springer: New York 2003.

[3] Canning J. Fresnel optics inside optical fibres. In Photonics research developments, Chapter 5. Nova Science Publishers, United States 2008.

[4] Nguyen H, Domachuk P, Eggleton B, et al. A new slant on photonic crystal fibers. Opt Exp 2004; 12: 1528-39.

[5] Martelli C, Canning J, Gibson B, Huntington S. Proceedings of the conference on the optical internet / Australian conference on optical fibre technology; July 2007; Engineers Australia; 2007.

[6] Sørensen HR, Canning J, Lægsgaard J, Hansen K, Varming P. Liquid filling of photonic crystal fibres for grating writing. Opt Comm 2007; 270(2): 207-10.

[7] Groothoff N, Canning J, Jovanovic N, Marshall GD, Withford MJ OSA Technical digest of Bragg gratings, photosensitivity, and poling in glass waveguides OSA 2007.

[8] Eggleton BJ, Krug PA, Poladian L, Ouellette F. Long periodic superstructure Bragg gratings in optical fibers. Electron Lett 1994; 30: $1620-2$.

[9] Sørensen HR, Deyerl H-J, Kristensen M. OSA Technical digest of Bragg gratings, photosensitivity, and poling in glass waveguides, OSA 2003.

[10] Cook K, Pohl AAP, Canning J. High-temperature type IIa gratings in 12-ring photonic crystal fibre with germanosilicate core. J Euro Opt Soc 2008; 3: 08031.

[11] Phan Huy MC, Laffont G, Frignac Y, et al. Fibre Bragg grating photowriting in microstructured optical fibres for refractive index measurement. Meas Sci Tech 2006; 17: 992-7.

[12] Canning J, Groothoff N, Buckley E, Ryan T, Lyytikainen K, Digweed J. All-fibre photonic crystal distributed Bragg reflector (PCDBR) fibre laser. Opt Exp 2003; 11: 1995-2000.

[13] Groothoff N, Canning J, Ryan T, Lyytikainen K, Inglis H. Distributed feedback photonic crystal fiber (DFB-PCF) laser. Opt Exp 2005; 13: 2924-30.

[14] Groothoff N, Canning J, Buckley E, Lyytikainen K, Zagari J. Bragg gratings in air-silica structured fibers. Opt Lett 2003; 28: 233-5.

[15] Groothoff N, Martelli C, Canning J, Lyytikainen K. Proceedings of Australian conference on optical fibre technology; EA Aust Sydney 2005.

[16] Mihailov SJ, Grobnic D, Huimin Ding, Smelser CW, Broeng J. Femtosecond IR laser fabrication of Bragg gratings in photonic crystal fibers and tapers. Phot Tech Lett 2006; 18: 1837-9.

[17] Neustruev, VB. Colour centres in germanosilicate glass and optical fibres. J Phys Cond Matt 1994; 6(35): 6901.

[18] Poumellec B, Douay M, Krupa JC, Garapon J, Niay P. Comparison of UV optical absorption and UV excited luminescence behaviours in Ge doped silica under $\mathrm{H}_{2}$ loading or $\mathrm{CW}$ UV laser irradiation. J N-Cryst Sol 2003; 317: 319-34.

[19] Marshall GD, Kan DJ, Asatryan AA, Botten LC, Withford MJ. Transverse coupling to the core of a photonic crystal fiber: the photo-inscription of gratings. Opt Exp 2007; 15: 7876-87.

[20] Geernaert T, Nasilowski T, Chah K, et al. Fiber Bragg gratings in germanium-doped highly birefringent microstructured optical fibers. Phot Tech Lett 2008: 20: 554-8.

[21] Groothoff N, Canning J. Enhanced type IIA gratings for high temperature operation. Opt Lett 2004; 29(20): 2360-2.

[22] Sceats M, Canning J. In: Limberger HG, Ed. Proceedings of summer school on photosensitivity in optical waveguides and glasses. Ecole Polytechnique Federale Lausanne, Lausanne; Switzerland: 1998. 